\title{
Responses in Macaque Visual Area V4 following Inactivation of the Parvocellular and Magnocellular LGN Pathways
}

\author{
Vincent P. Ferrera, Tara A. Nealey, and John H. R. Maunsell \\ Department of Physiology and Center for Visual Science, University of Rochester, Rochester, New York 14642-8642
}

\begin{abstract}
A substantial body of evidence has suggested that signals transmitted through the magnocellular and parvocellular subdivisions of the LGN remain largely segregated in visual cortex. This hypothesis can be tested directly by selectively blocking transmission through either the magnocellular or parvocellular layers with small injections of lidocaine or GABA while recording cortical responses to a visual stimulus. In a previous study, we found that responses in the middle temporal visual area (MT) were almost always greatly reduced by blocks of magnocellular LGN, but that few MT neurons were affected by parvocellular blocks. In the present study, we have examined magnocellular and parvocellular contributions to area $V 4$, which lies at the same level of processing in the cortical hierarchy as does MT and is thought to be a major recipient of parvocellular input. We found that inactivation of parvocellular layers usually resulted in a moderate reduction of visual responses (median reduction, $36 \%$ ). However, comparable reductions in V4 responses were also seen following magnocellular blocks (median reduction, $47 \%$ ). Directionally selective responses in V4 were not found to depend specifically on either subdivision. We conclude that area $\mathrm{V4}$, unlike $M T$, receives strong input from both subdivisions of the LGN. These results suggest that the relationship between the subcortical magnocellular and parvocellular pathways and the parietal and temporal streams of processing in cortex is not one-to-one.
\end{abstract}

[Key words: V4, LGN, magnocellular, parvocellular, macaque monkey, extrastriate]

The visual cerebral cortex of primates contains two streams of processing that include different extrastriate visual areas and contribute to different types of visual behaviors. The parietal stream is associated with more dorsal extrastriate visual areas, including the middle temporal visual area (MT) and much of parietal cortex. It is thought to be involved in the analysis of motion and spatial orientation, and to be important for visual guidance. The temporal stream comprises more ventral areas including V4 and inferotemporal cortex. It is involved in color, form, and object identification (Ungerleider and Mishkin, 1982: Mishkin et al., 1983; Van Essen and Maunsell, 1983; Van Essen,

Received June 29, 1993; revised Sept. 9, 1993; accepted Sept. 21, 1993

We thank Mr. Peter Vamvakias for excellent technical assistance, and John Assad, Daniel Felleman. Nikos Logothetis, and Kirsten Rudolph for critical comments on an early version of the manuscript. This research was supported by NIH R01 EY05911 and Postdoctoral Fellowship NS08658 10 V.P.F. and a McKnight Foundation Development Award.

Correspondence should be addressed to John MaunselI. Division of Neuroscience, S-603, Baylor College of Medicine, One Baylor Plaza, Houston, TX 77030. Copyright (c) 1994 Society for Neuroscience $0270-6474 / 94 / 142080-09 \$ 05.00 / 0$
1985). While the separation of the parietal and temporal streams is not complete, they appear to maintain appreciable anatomical segregation and possess markedly different neuronal response properties (see Merigan and Maunsell, 1993).

Because the parietal and temporal streams of processing represent a major subdivision of visual cortex, they offer one of the best opportunities for approaching the origins and significance of functional specialization in cortex. One basic question concerns the origins of the differences in the response properties of their neurons. Neurons in the parietal stream are highly selective for the direction of stimulus motion, but relatively insensitive to color, size, or form. In contrast, visual areas in the temporal pathway do not usually show strong direction selectivity and are instead more selective for color, orientation, or form. It has been suggested that these physiological differences may depend largely on differential contributions from the magnocellular (M) and parvocellular (P) pathways, such that the parietal pathway receives input from the magnocellular LGN, whereas the parvocellular LGN contributes primarily to the temporal pathway (Livingstone and Hubel, 1987a, 1988; Maunscll, 1987).

Many converging lines of evidence have suggested that magnocellular and parvocellular contributions could be kept sepa rate in the early stages of visual cortex and directed selectively to the parietal and temporal streams (see I ivingstone and Hubel, 1987a, 1988; Maunsell, 1987; Maunsell and Newsome, 1987 . DeYoe and Van Essen, 1988; Martin, 1988; Desimone and Ungerleider, 1989; Felleman and Van Essen, 1991; Kaas and Garraghty, 1991). Furthermore, experiments using selective inactivation of LGN subdivisions have shown that neurons in MT, an important member of the parietal stream, receive most of their excitatory drive from the magnocellular subdivision of the LGN (Maunsell et al., 1990). It has not yet been established whether a corresponding parvocellular dominance exists in the temporal pathway. Recent anatomical and physiological evidence suggests that $\mathrm{M}$ and $\mathrm{P}$ pathway contributions mix considerably in the superficial layers of V 1 , which provide the major in put to the temporal stream of processing (Lachica et al., 1992; Nealey and Maunsell, 1994).

Despite indications that both $M$ and $P$ pathway contributions may reach extrastriate areas in the temporal pathway, it need not be the case that they do. Relatively pure $M$ pathway signals survive the mixing that occurs in V1 and project to the superior colliculus (Schiller et al., 1979) and MT (Maunsell et al., 1990). The connections leading to the temporal stream might correspondingly receive a relatively pure $P$ pathway signal. To understand the relationship between the subcortical pathways and the temporal stream of processing more completely, we have examined magnocellular and parvocellular contributions to vi- 


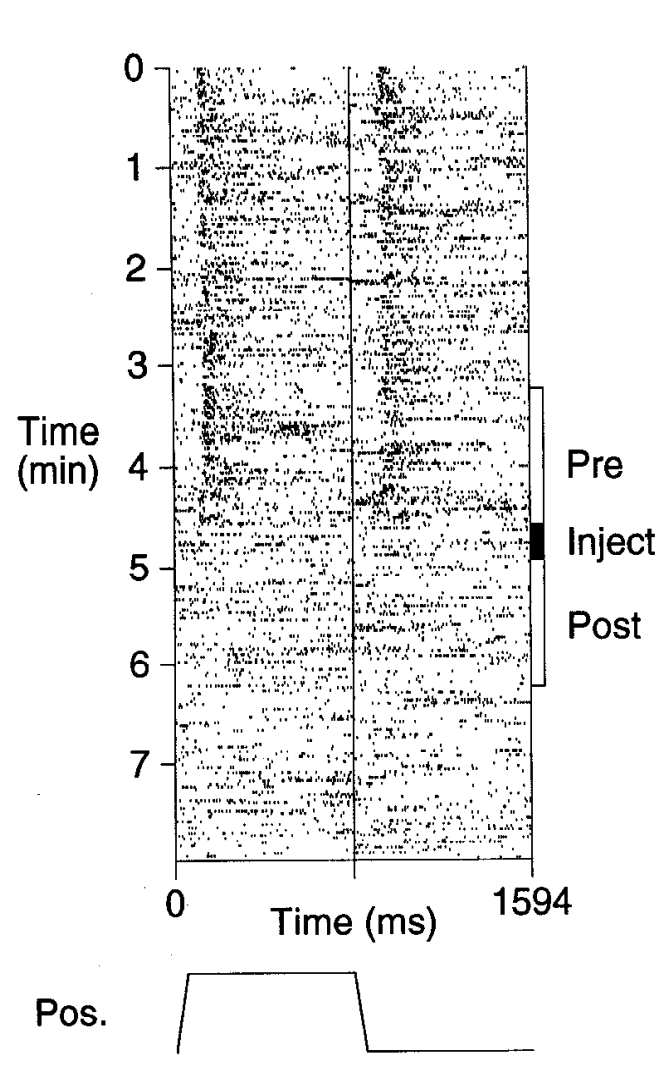

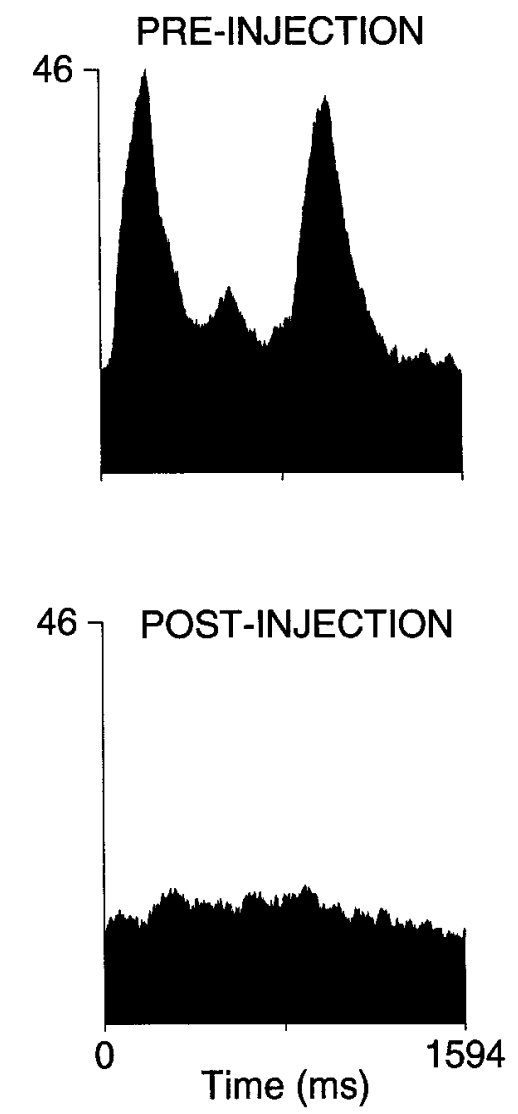

Figure 1. Effect of magnocellular LGN block on responses in V4. The left panel shows the responses of a multiunit V4 site before and after an injection of 100 $\mathrm{nl}$ of lidocaine into layer 1 of the LGN. Time increases going downward, with each horizontal raster line representing one stimulus cycle. Each stimulus cycle lasted about $1.5 \mathrm{sec}$ and consisted of the movement of a small bar back and forth along the preferred axis with a long pause before each change of direction. The line at the bottom of the raster plots position as a function of time. The response of the unit was abolished shortly after the onset of the injection. The period during which the injection was made is marked by a solid bar on the right side of the raster (Inject). The right panels show smoothed average histograms of responses collected immediately before and after the injection. Each contains 50 stimulus cycles, taken from the periods marked with the open bars to the right of the raster (Pre and Post). Background activity was also measured for the equivalent of 50 trials with no stimulus present (not shown). The vertical axes are scaled in impulses $/ \mathrm{sec}$. The preinjection response (peak - trough minus the background peak/trough difference) was $24.3 \mathrm{impulses} / \mathrm{sec}$, while the postinjection response was -4.5 impulses/sec, yielding a BI of 1.19 . sual responses in area V4 by selectively inactivating one pathway. We chose to look at area V4 because it occupies a position in the temporal stream that is at the same hierarchical level as MT (Felleman and Van Essen, 1991). V4 was first described by Zeki $(1971,1973)$, who noted that it contains many neurons that are color selective. Subsequent studies have documented other properties of $\mathrm{V} 4$ neurons, including sensitivity for stimulus orientation, length, and width (Zeki, 1978a; Desimone and Schein, 1987). Wc have found that both subdivisions of the LGN make a substantial contribution to visual responses in V4. These results do not support the idea that visual cortex in the temporal lobe is dominated by parvocellular input.

Some of the data have been described previously in a short report (Ferrera et al., 1992).

\section{Materials and Methods}

The methods of data collection and analysis have been described in detail elsewhere (Maunsell et al., 1990; Nealey and Maunsell, 1994). Data were collected from anesthetized, paralyzed macaque monkeys. Recordings were made in five animals (two Macaca fascicularis and three $M$. nemestrina) that weighed between 2 and $7 \mathrm{~kg}$. Selective LGN inactivation (Malpeli and Schiller, 1979) was achieved using recording injecting probes (Maunsell et al., 1990) that were loaded with a solution of either $2 \%$ lidocaine or $25 \mathrm{~mm} \gamma$-aminobutyric acid (GABA) in isotonic saline. Either single- or multiple unit recordings were made from cortex during data collection. Multiunit recording sites were separated by at least $250 \mu \mathrm{m}$.

Injection volumes for lidocaine or GABA were in the range of 25$250 \mathrm{nl}$, with most injections between 50 and $150 \mathrm{nl}$. We have found previously (Maunsell et al., 1990) that for this range of injection volumes there was no correlation between the amount of blocking agent injected and the magnitude of the effect on cortical responses, indicating that the injections were effective at blocking the entire representation of the visual stimulus in the LGN. The range of effects for these agents is restricted to about $1 \mathrm{~mm}$ from the injection center (Maunsell et al., 1990; Nealey and Maunsell, 1994). In a few experiments we also checked that blocking agents did not spread between LGN subdivisions by making injections in the parvocellular subdivision of the LGN while recording simultaneously from corresponding visual field representations in both V4 and the noninjected magnocellular subdivision. These recordings confirmed that the effects of the blocking agent were confined to the region of the injected layer (see Results).

Cortical responses were driven using only the contralateral eye. Using this approach, the only LGN layers that were active were 1, 4, and 6 , and the physical separation between active layers made it possible to confine the effects of injection to one LGN subdivision (Nealey and Maunsell, 1994). Because most units in V4 respond readily to either eye (Zeki, 1978b), monocular stimulation does not substantially reduce cortical responses. The receptive fields found in $\mathrm{V} 4$ cover regions of the visual field that are too extensive to be inactivated with small LGN injections. However, most neurons in V4 respond well to small targets that flash on or off or move back and forth along a short excursion within a portion of the visual field that can be inactivated by an LGN injection. In these experiments $\mathrm{V} 4$ responses were driven with visual stimuli that never traveled more than $2^{\circ}$ from the ecnter of the blocked LGN representation. We located receptive fields in V4 that were centered away from the fovea (average eccentricity, $5.9^{\circ}$ ), so that corresponding representations in the LGN had moderate to low magnification (e.g., magnification at $6^{\circ}$ in LGN layer 6 is $\sim 320 \mu \mathrm{m} /$ degree and in LGN layer 1 is $\sim 160 \mu \mathrm{m} /$ degree; Connolly and Van Essen, 1984). With this approach the entire representation of a visual stimulus within an LGN layer could be inactivated with a single injection. Once receptive fields in V4 and the LGN were suitably aligned and an adequate visual stimulus was found, data were collected while the stimulus was presented under computer control. Moving stimuli swept back and forth across the receptive field in the preferred and null directions at the site's preferred speed and orientation.

Changes in cortical response following LGN inactivation were quantified using a blocking index (BI) (Maunsell et al., 1990; Nealey and Maunsell, 1994). Responses immediately before or after the injection 


\section{MAGNOCELLULAR BLOCKS}

A: Bl 0.13
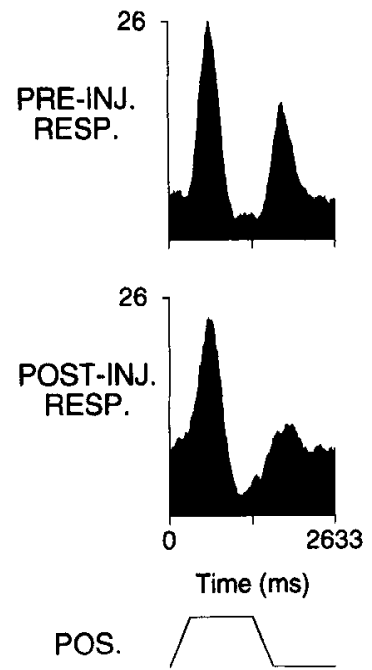

$\mathrm{B}: \mathrm{Bl} 0.48$
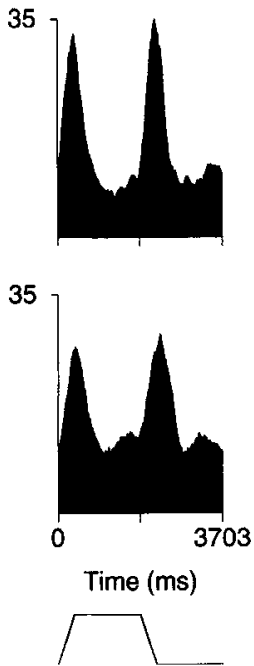

C: BI 0.75

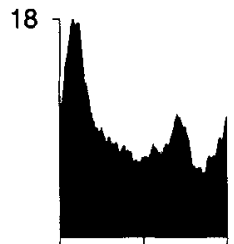

18

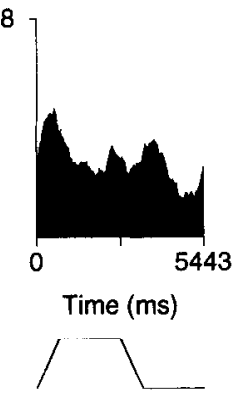

Figure 2. Effects of magnocellular blocks for three representative multiunit V4 sites. Each pair of histograms shows responses before and after blocking LGN layer 1, and were prepared as in Figure 1. The blocking indices $(B I)$ for these sites were close to the 25 th, 50 th, and 75 th percentiles for the distribution of magnocellular blocking indices. Injections: $A, 100$ nl of tiducaine; $B$ and $C, 100$ nl or GABA. The vertical axes are scaled in impulses/sec. The lines below the histograms indicate the stimuli all moved back and forth with a pause between each change of direction.

were computed by averaging 50 stimulus cycles and smoothing the resulting histograms with a boxcar average (Fig. 1). An averaging length of $1 / 10$ of the stimulus cycle was used. Response was based on the difference between the peak and trough in each histogram so that both excitatory and inhibitory effects were detected. To compensate for peak trough differences resulting from noise, the stimulus response was taken to be the peak/trough difference in the response histogram minus the peak/trough difference determined from activity recorded in the absence of a stimulus. The blocking index was taken as $1.0-$ (postinjection response/preinjection response). A value of 0 represents no block and 1 represents no response following the block. This blocking index is negative when the response increases following LGN block, and can exceed 1.0 if the difference between the peak and trough in the postinjection histogram was less than the peak/trough difference in the absence of a visual stimulus.

At the end of the recording session the animal was given an overdose of barbiturates and perfused with a phosphate-buffered saline rinse $(\mathrm{pH}$ 7.4) followed by paraformaldehyde fixative. The brain was removed blocked, and equilibrated with $30 \%$ sucrose in phosphate buffer. Blocks were then sectioned at a thickness of $40 \mu \mathrm{m}$. Series of sections were stained for Nissl substance and myelin (Gallyas, 1979). Electrode tracks and electrolytic lesions were marked on enlargements of the histological sections. Each cortical recording site was located based on microdrive readings and distance relative to lesions and cortical borders. Recording sites were assigned to $\mathrm{V} 4$ based on its position on the prelunate gyrus relative to MT, which was identified by its distinctive myeloarchitectonics (Van Essen et al., 1981). Electrode penetrations within the LGN were not reconstructed.

\section{Results}

\section{Effects of blocking magnocellular $L G N$}

We found a substantial magnocellular contribution to V4 responses. Over one-third of the $51 \mathrm{~V} 4$ sites tested showed a $50 \%$ or greater reduction in response when magnocellular inputs were blocked. In several cases, responses were essentially eliminated. Figure 1 shows activity recorded from a multiunit site in V4
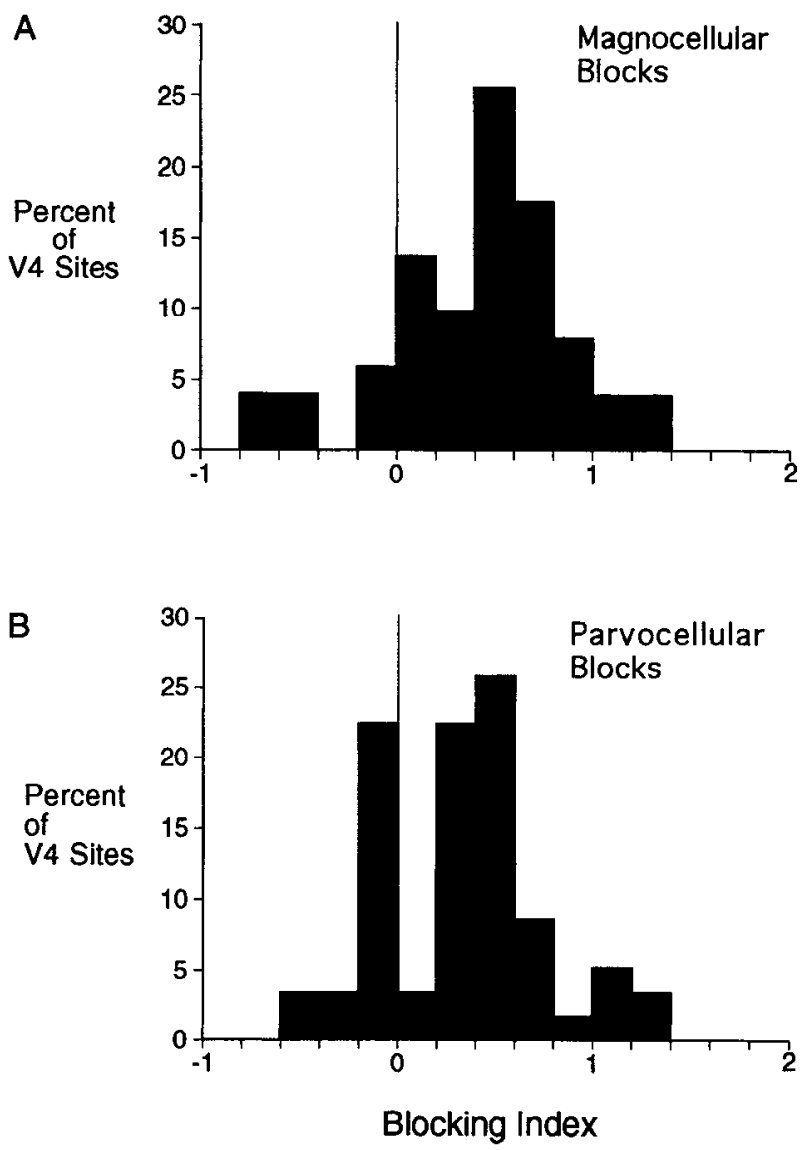

Figure 3. Distribution of magnocellular and parvocellular BIs. The median of the distribution for magnocellular blocks $(0.47)$ was not significantly different than the parvocellular median (0.36). Some V4 sites werc testcd for contributions from layers 4 and 6 individually. In those cases the average of the two BIs was used.

before and after lidocaine was injected into magnocellular layer 1. The stimulus was a bar moving back and forth over a short excursion, and the V4 site responded to both directions of motion until the time of injection, at which point the response was eliminated. The BI for this case was 1.19 , indicating that the postinjection response was slightly less than the random fluctuations in activity measured during the background period.

Figure 2 illustrates the range of effects that was found with magnocellular blocks. As in Figure 1, each pair of histograms shows responses averaged over 50 stimulus cycles before and immediately following the injections. The middle pair of histograms (Fig. $2 B$ ) shows the effects of an injection that produced a blocking index near the median value of the distribution for all magnocellular injections. The sites whose responses appear in Figure 2, $A$ and $C$, had blocking indices near the 25th and 75 th percentiles. The effect of the block is pronounced in Figure $2 C$, and more subtle, but still obvious, in Figure $2, A$ and $B$.

The distribution of blocking indices from magnocellular injections is shown in Figure $3 A$. The median of the magnocellular BIs is 0.47 , which corresponds to nearly halving the response. This value is slightly greater than the median magnocellular BI found in the superficial layers of V1, which was 0.37 (Nealey and Maunsell, 1994). About 10\% (5 of 51) of the V4 sites had responses that were effectively eliminated (BI $\geq 1.0$ ) by magnocellular blockade. BIs greater than 1.0 indicate that postin- 
PARVOCELLULAR BLOCKS
A: $\mathrm{BI}-0.08$
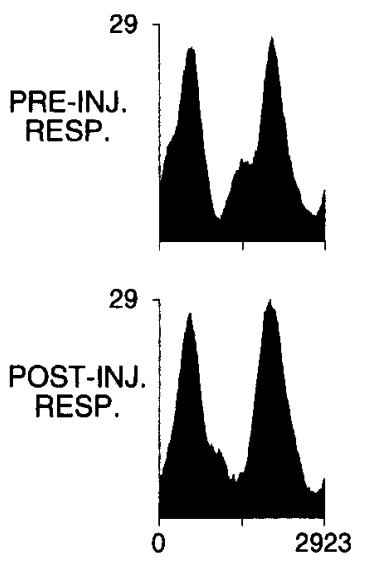

POS.
B: BI 0.36

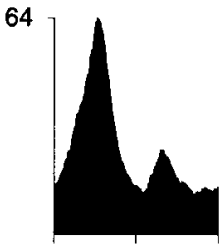

64

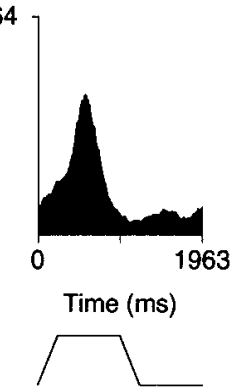

C: BI 0.55

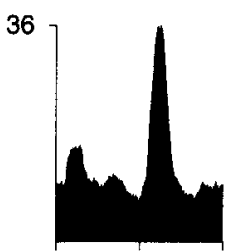

36

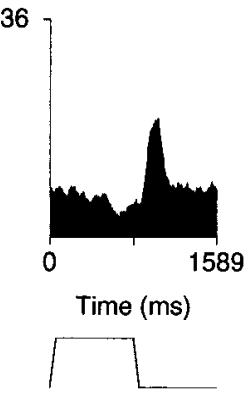

Figure 4. Parvocellular blocks for three representative multiunit V4 sites. Each pair of histograms shows V4 responses before and after block of one parvocellular LGN layer. The blocking indices shown are close to the values of the $25 \mathrm{th}$, 50th, and 75th percentiles of the distribution of parvocellular indices. Injections: $A, 100 \mathrm{nl}$ of GABA into layer $6 ; B$, $90 \mathrm{nl}$ of lidocaine into layer $4 ; C, 60$ nl of lidocaine into layer 6 . The vertical axes are scaled in impulses/sec. The lines below the histograms indicate the stimuli all moved back and forth with a pause between each change of direction.

jection response was less than the random fluctuations in activity that occurred in the absence of stimulation. Values less than zero indicate that the response grew stronger after the injection was made. Some small negative BIs are probably due to random fluctuations in cortical responsiveness. Large negative BIs were relatively rare, but suggest that some V4 sites receive inhibitory input from the magnocellular channel.

There was no significant difference in the effects of magnocellular blocks on single units (median BI, $0.23 ; n=13$ ) versus multiunit recording sites (median $\mathrm{BI}, 0.47 ; n=38$; Mann-Whitney test, $p=0.13$ ). There was also no difference between the effects of magnocellular blocks produced with lidocaine (median BI, $0.47 ; n=19$ ) or GABA (median BI, $0.47 ; n=32$; MannWhitney test, $p=0.76$ ).

\section{Effects of blocking parvocellular $L G N$}

Parvocellular block reduced cortical responses by an amount similar to the effects of magnocellular block. The distributions of blocking indices for parvocellular and magnocellular blocks are compared in Figure 3. The median of the parvocellular BIs $(0.36, n=58)$ is not significantly different than the magnocellular median (Mann-Whitney test, $p=0.25$ ). This value is also close to the median parvocellular $\mathrm{BI}$ found in the superficial layers of V1, which was 0.39 (Nealey and Maunsell, 1994). Many parvocellular injections failed to have any discernible effect on $\checkmark 4$ responses.

The range of blocking effects seen following parvocellular blocks is shown in Figure 4. As in Figure 2, the BIs for the threc sites shown are close to the 25 th, 50 th, and 75 th percentiles. There was no significant difference between single units (median $\mathrm{BI}, 0.54 ; n=13$ ) and multiunit sites (median BI, $0.34 ; n=45$;

\section{Complete Parvo Block $\mathrm{Bl}: 0.13$}
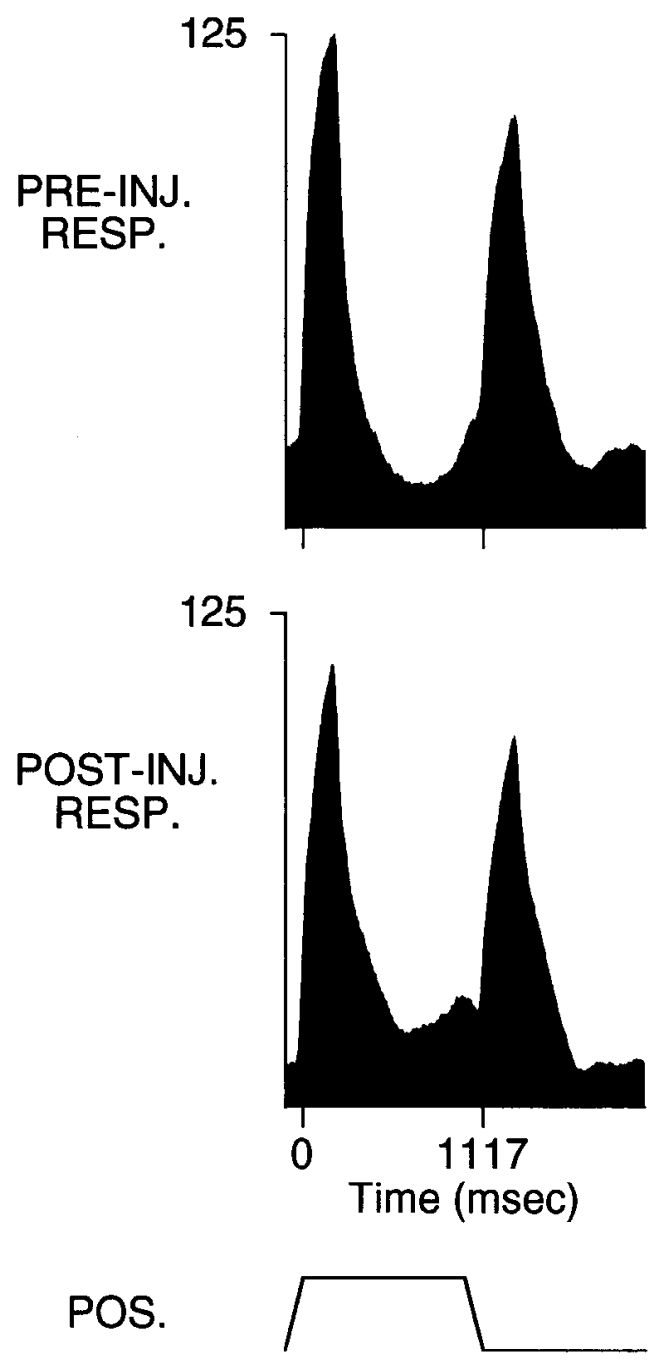

Figure 5. Effects of a complete parvocellular block on V4 responses. Responses were recorded from a small multiunit cluster while GABA injections were made simultaneously into parvocellular layers 4 and 6 , thereby silencing all parvocellular contributions. The upper histogram shows V4 response immediately before the injections, and the lower histogram shows responses immediately after. While there was a slight reduction in response, the magnocellular contributions were able to support almost all of the responses at this site.

Mann-Whitney test, $p=0.20$ ), or between the effects of lidocaine (median BI, $0.40 ; n=28$ ) and GABA (median BI, 0.27; $n=30 ;$ Mann-Whitney test, $p=0.46$ ).

Data were collected using monocular stimuli that activated two parvocellular layers and one magnocellular layer. A single parvocellular layer was blocked by each injection, leaving open the possibility that responses might be mediated by the unblocked parvocellular layer. In studies of other visual areas it has been possible to examine the effect of completely removing parvocellular input by recording in regions representing eccentric parts of the visual field (Maunsell et al., 1990; Nealey and Maunsell, 1994). At large cccentricities only a single parvocellular layer is driven by the contralateral eye, and this contribution can be silenced with a single injection. This approach was not practical in the case of V4 because the topographic 


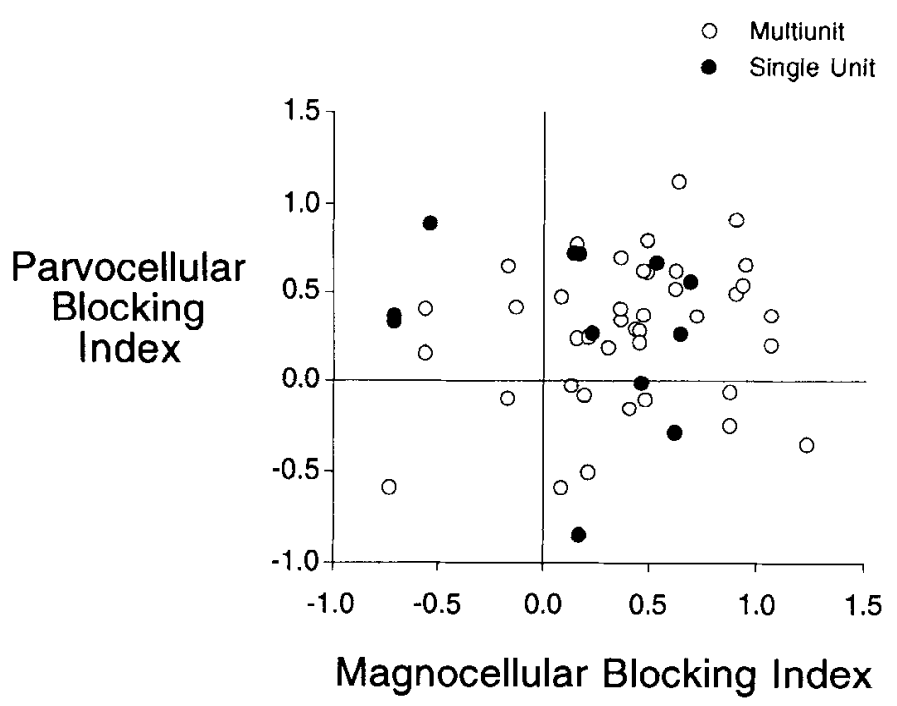

Figure 6. Relationship between magnocellular BI and parvocellular BI for individual sites tested with a layer 1 injection and either a layer 4 or layer 6 injection. In cases where both parvocellular layers were tested, only the layer with the larger BI is shown. Open circles are multiunit V4 recording sites and solid circles are single units.

representation of eccentric regions is not orderly and has not becn adequately characterized to permit certainty about whether a given site is actually within V4 (see Van Essen, 1985). As an alternative, in one animal we placed two probes in the I GN so that injections could be made simultaneously in layers 6 and 4 . Complete parvocellular block did not eliminate responses at the four V4 sites tested this way. Figure 5 shows the effects of one of the paired LGN injections on the responses of a V4 site. Completely removing all parvocellular contributions resulted in only a slight reduction in response (BI, 0.13 ). The largest BI for the four sites was 0.85 . While only a handful of sites were examined with complete parvocellular blocks, the results of those tests show that magnocellular inputs alone can support responses in V4.

\section{Magnocellular and parvocellular contributions to individual sites in $\mathrm{V} 4$}

The results presented above suggest that both subdivisions of the LGN contribute to responses in area V4. It is possible, however, that the magnocellular and parvocellular inputs to V4 have a patchy distribution that maintains the segregation of the two subcortical pathways. Were this the case, one would expect to find sites that receive input almost exclusively from one pathway, the inactivation of which would produce a complete block like that shown in Figure 1. However, this result was relatively uncommon. Thirty-eight sites in V4, including 11 single units, were tested first for contributions from one subdivision of the LGN and then from the other. Figure 6 shows the relationship between magnocellular and parvocellular BIs at individual V4 sites. Most sites are clustered in a region corresponding to moderate effects of blocking either pathway.

When we looked more closely at sites for which all three active layers had been blocked individually $(n=14)$, only two sites appeared to be dominated by the $P$ pathway and one by the $M$ pathway, such that the response virtually disappeared when one pathway was blocked but was unaffected when the other pathway was blocked. The data in Figure 7 illustrate the more common
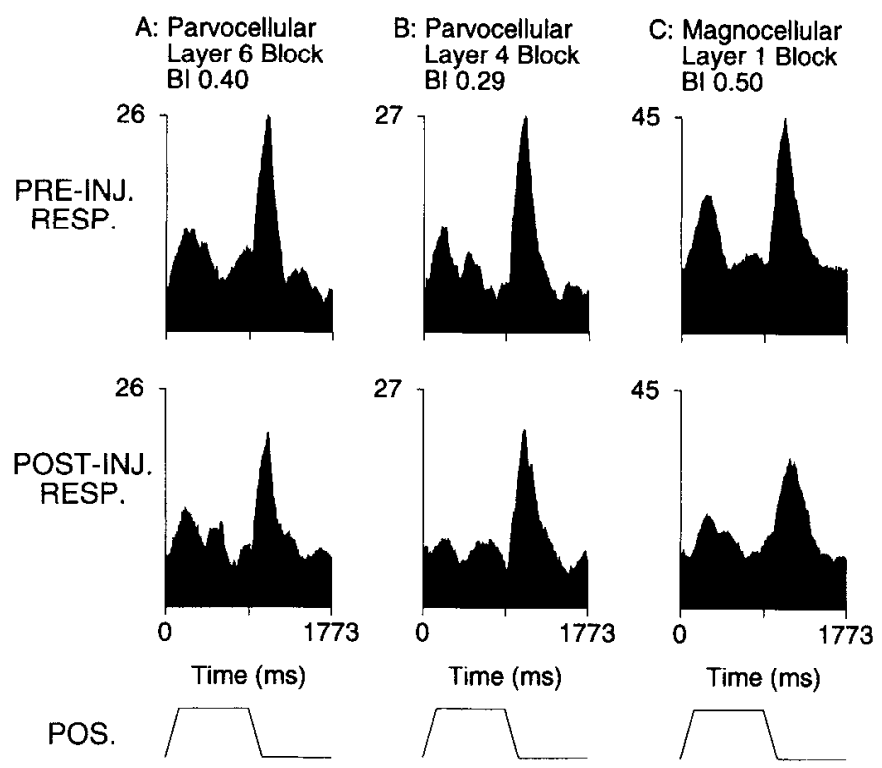

Figure 7. Responses from a V4 multiunit site that was tested with blocks of cach of the three active LGN layers. Each layer was blocked individually while recording from the same V4 site. Each block resulted in a partial reduction of cortical response. It was typical to find that V4 multiunit sites and isolated units received demonstrable inputs from both subdivisions and all three layers.

result. The figure shows pre- and postinjection responses from a multiunit site in V4 that was tested while layers 6,4 , and 1 were blocked one after the next. Silencing any of the three layers reduced but did not eliminate responses. This and other sites demonstrate that magnocellular and parvocellular contributions are not strongly segregated into different regions of V4.

Even though magnocellular and parvocellular inputs to area V4 seem to be homogeneously distributed, it remained possible that single neurons might receive input exclusivcly from onc pathway or the other. Data from 11 single units tested for both magnocellular and parvocellular contributions argue against this idea. Only one unit was completely blocked by a parvocellular injection and none by magnocellular injections. Most units had only a partial reduction in response when either pathway was inactivated. Figure 8 shows the responses of a single unit in V4 tested with both types of LGN block. The magnocellular block produced a partial reduction in the unit's response, and this effect was indistinguishable from the effect of a parvocellular block. Thus, while some units in V4 receive exclusively magnocellular or parvocellular input, the majority seem to receive mixed inputs.

\section{Relationship between direction selectivity and geniculate input}

All cortical sites in V4 were tested with moving stimuli, allowing us to determine the directional selectivity of the response before and after blocking the subcortical input. As expected, direction selectivity in V4 was generally weak. Selectivity was quantified using the formula $\left(R_{\text {pref }}-R_{\text {null }}\right) /\left(R_{\text {pref }}+R_{\text {null }}\right)$, where $R$ indicates response in the preferred or null direction, computed as for the BI. This yields a direction index varying between 0 and 1 . A direction index of 0.33 corresponds to a $2: 1$ ratio between the responses to the preferred and null dircetions, whereas a direction index of 0.67 corresponds to a $5: 1$ ratio. The median direction index for the baseline response at 72 sites was 0.17 (1.4: 1). Seventeen sites $(24 \%)$ had a preferred: null ratio of $2: 1$ or 

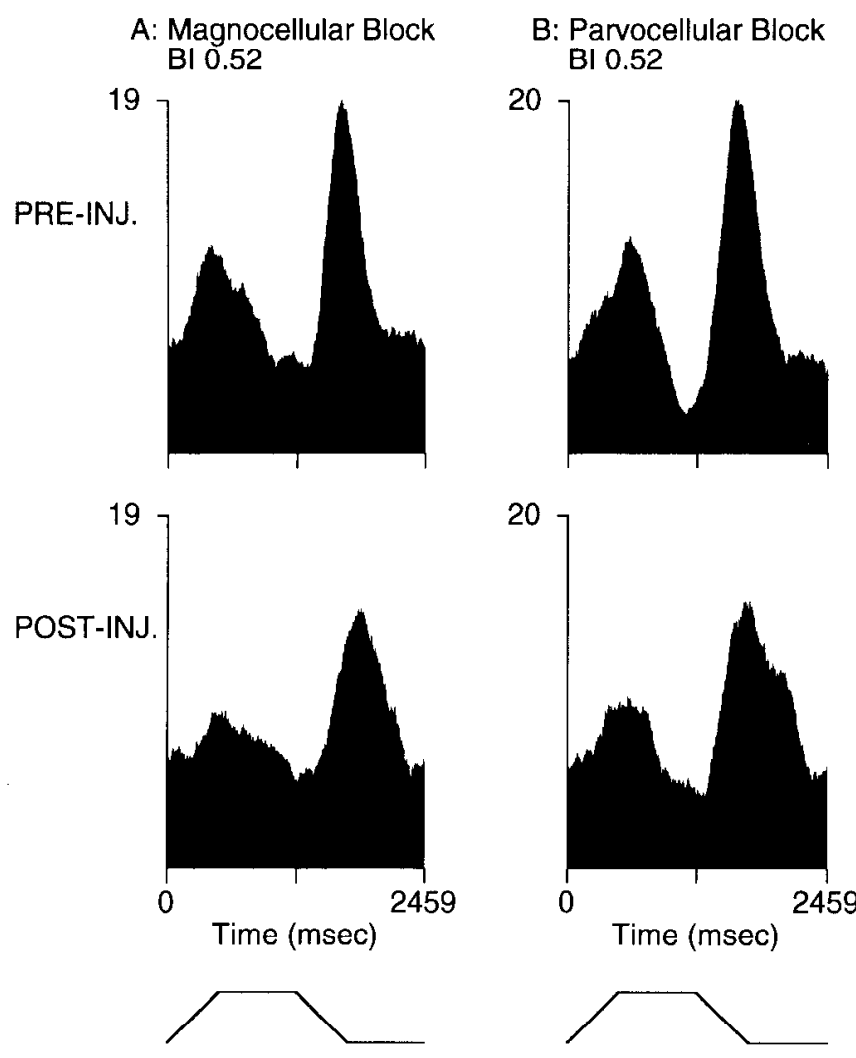

Figure 8. Mixed magnocellular and parvocellular contributions to a single unit in V4. $A$, Effect of a $100 \mathrm{nl}$ injection of GABA into LGN layer $1(\mathrm{BI}, 0.52)$. B, Effect of a $100 \mathrm{nl}$ injection of GABA into LGN layer $6(\mathrm{BI}, 0.52)$.

greater, and only one site had a ratio greater than $5: 1$. The median direction index for single units was 0.21 .

Direction selectivity was not a reliable predictor of magnocellular or parvocellular contributions to any given site. There was no significant correlation between the magnitude of the direction index (measured before the injection) and the blocking index for either magnocellular $(r=0.02, p=0.90)$ or parvocellular injections $(r=0.12, p=0.26)$. Thus, there was no tendency for sites with greater direction selectivity to receive a stronger magnoccllular contribution.

Furthermore, direction selectivity was not significantly altered by injections of either kind. Following parvocellular injections, the median direction index went from 0.13 to 0.17 (MannWhitney test, $p=0.25$ ), whereas following magnocellular injections the median went from 0.14 to $0.12(p=0.78)$. The changes in directionality for single units were likewise not significant. In general, direction selectivity was not affected by changes in the overall response produced by blocking geniculate inputs, nor was there a tendency for direction selectivity to be associated with contributions from one pathway or the other. This general tendency for V4 sites to retain their overall response profile following selective LGN inactivation can be seen in the histograms presented in the figures. These results support the idea that direction selectivity is determined by cortical processing that is not dependent on a particular subcortical pathway. This observation is consistent with the report of Malpeli et al. (1981), who found that orientation and direction selectivity in macaque V1 did not depend exclusively on either LGN subdivision.
MAGNOCELLULAR

BLOCK
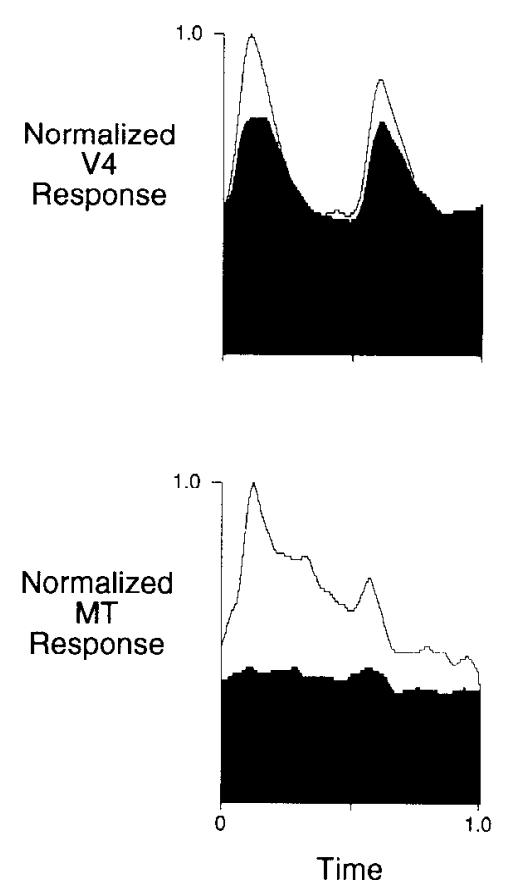

Figure 9. Composite preinjection (white) and postinjection (black) histograms obtained by pooling responses over all $V 4$ recording sites and for corresponding data collected from MT in an earlier study (Maunsell et al., 1990). The pre- and postinjection response histograms for each site were first normalized to the same peak preinjection response and a time of 1.0 stimulus cycle. If the peak in the normalized preinjection histogram was during the second half-cycle, the histogram was shifted by one half-cycle to place the peak in the first half. The normalized histograms for each injection type were then averaged together. The resulting composite histograms were smoothed by convolving with a unit Gaussian that had a standard deviation equal to 0.02 stimulus cycles. Blocking indices were computed for both peaks in both sets of histograms. The average of the two magnocellular BIs for V4 was 0.37 , while the parvocellular average was 0.29 . For MT, the average of the two magnocellular BIs was 0.76 , while the parvoccllular average was 0.04 .

\section{$P$ and $M$ contributions to $V 4$ and $M T$}

To characterize the overall effects of LGN blockade on visual responses, we pooled the responses from all recording sites into composite pre- and postinjection histograms (see Fig. 9 caption for details). The results are shown in Figure 9, in which data from V4 are plotted in the upper row. The white histograms are the average response before block, and the black histograms show the responses following $\mathrm{M}$ or $\mathrm{P}$ pathway block. These composite response histograms show that both $\mathrm{M}$ and $\mathrm{P}$ pathway blocks caused moderate reductions in V4 responses. They also show the absence of a pronounced change in direction selectivity.

Similar composite histograms were compiled for responses recorded previously from MT (Maunsell et al., 1990) and are shown in the lower row of Figure 9. The greater direction selectivity of MT neurons is readily seen in the preinjection responses (white histograms). The effects of LGN blocks on MT responses are strikingly different from the pattern of results for V4. As reported earlier, magnocellular injections left little response in MT (average magnocellular BI, 0.72), whereas par- 
vocellular injections had little effect (average BI, 0.11). Taken together, the data of Figure 9 show that the striking asymmetry in the effects of magnocellular and parvocellular LGN blocks on MT responses is not seen in area V4, where the two LGN pathways provide more balanced, moderate contributions.

\section{Discussion}

These results show that the $M$ and $P$ subcortical pathways both contribute to visual responses in V4, an important stage of processing in the temporal stream of processing. The method of selective LGN inactivation does not permit precise statements about the strength of the contributions (see Maunsell et al., 1990), but the results suggest that magnocellular contributions are at least as important as parvocellular contributions under normal viewing conditions. There was no evidence that magnocellular and parvocellular pathways are appreciably segregated within V4; most sites receive contributions from both pathways. It is therefore likely that visual areas in inferotemporal cortex, which receives a major input from V4, also have mixed magnocellular and parvocellular contributions.

\section{$P$ and $M$ pathway segregation within $V 4$}

We saw little evidence that magnocellular and parvocellular inputs were segregated within V4. Most multiunit and singleunit recording sites showed moderate reductions in response when either LGN pathway was blocked. Anatomical evidence suggests that the route of input to V4 that is provided by the $\mathrm{V} 1$ blobs and the $\mathrm{V} 2$ thin stripes stays segregated from the route of input provided by the V1 interblobs and the V2 pale stripes, and that regions of $\mathrm{V} 4$ several millimeters across are dominated by one or the other route (DeYoe and Sesola, 1991; Felleman and McClendon, 1991). Although there is no compelling evidence for $\mathrm{P}$ and $\mathrm{M}$ pathway segregation between the blobs and interblobs (Ncalcy and Maunsell, 1994), it was possible that mechanisms might act to enhance a slight segregation at later stages. Although we had no anatomical marker for regions receiving inputs from the pale stripes and thin stripes, both magnocellular and parvocellular contributions could be demonstrated at most of the V4 sites tested. Thus, neither of the V4 regions that receive input from the two routes defined by cytochrome oxidase compartments appears to be associated with either a pure magnocellular or pure parvocellular subsystem within V4. While it is possible that our sampling of V4 missed regions that receive predominantly one type of input (e.g., regions receiving projections from MT or V3), the failure to find any clear segregation of magnocellular and parvocellular inputs in V4 suggests that the intermixing of $P$ and $M$ pathway contributions in the blobs and the interblobs in V1 persists throughout the temporal stream.

Another, coarser, segregation has been demonstrated in V4. Baizer et al. (1991) showed that while large portions of V4 project primarily to inferotemporal cortex, neurons in more dorsal portions of V4 also project to parietal cortex. Most of our recording sites had receptive fields in the central $10^{\circ}$ of the visual field (average eccentricity, $5.9^{\circ}$ ), and it is therefore likely that we recorded from parts of V4 that provide input to inferotemporal cortex, but not to posterior parietal cortex. It is possible that the LGN contributions to more dorsal portions of V4 would be more strongly dominated by magnocellular contributions.

\section{$P$ and $M$ pathway contributions to $\mathrm{V} 4$ and $M T$}

We chose to look at V4 because it lies at the same hierarchical level in the macaque's visual cortcx as docs MT (Felleman and Van Essen, 1991). MT is an important early stage of the parietal stream, whereas V4 is thought to have a comparable position in the temporal stream. Comparison of the $M$ and $P$ pathway contributions in V4 with corresponding data from MT (Maunsell et al., 1990) reveals an asymmetry between the parietal and temporal streams. MT was found to depend primarily on magnocellular input, although some sites within it showed minor effects of parvocellular blockade. The dominance of $\mathrm{M}$ pathway input in MT is in sharp contrast to the weaker and more even effects seen in V4 (Fig. 9).

Some caution is needed in comparing the results for MT and V4. Unlike the $P$ pathway blocks made in testing MT, those used for V4 left one parvocellular layer active, and are likely to have underestimated total $P$ pathway contribution. It is therefore possible that the $P$ pathway makes a larger contribution to responses in V4 than is indicated by our data. Nevertheless, it is clear that there is an asymmetry between the parietal and temporal streams in that the $M$ pathway contribution to V4 is more pronounced than the $\mathrm{P}$ pathway contribution to $\mathrm{MT}$. It is possible that $\mathrm{M}$ and $\mathrm{P}$ pathway contributions are more thoroughly mixed at later stages of the parietal stream, but there is little reason to suspect that this is the case given what is known about the physiology and connections of the parietal stream. At the very least mixing is more complete at an earlier stage in the temporal stream than in the parietal stream.

This asymmetry in the relationships between the $M$ and $P$ pathways and the parietal and temporal streams is consistent with known anatomical connections. MT receives a major input from layer 4B in V1, both directly (Lund et al., 1976) and via the thick cytochrome oxidase stripes in V2 (DeYoe and Van Essen, 1985; Shipp and Zeki, 1985; Livingstone and Hubel, $1987 \mathrm{~b}$ ). Layer $4 \mathrm{~B}$ is known to receive a strong input from layer $4 \mathrm{C} \alpha$, where the magnocellular LGN projection terminates. There is little indication of a strong parvocellular influence in layer $4 \mathrm{~B}$ : the parvocellular inputs to layer $4 \mathrm{C} \beta$ and $4 \mathrm{~A}$ are relayed primarily to more superficial layers (Lund and Boothe, 1975; Lund et al., 1979). On the other hand, V4 receives indirect input from all portions of the superficial layers of V1 (Livingstone and $\mathrm{Hubcl}, 1984)$. In addition to the parvocellular contributions mentioned above, the superficial layers also receive substantial projections from layers dominated by magnocellular inputs (Blasdel et al., 1985; Fitzpatrick et al., 1985; Lachica et al., 1992). Thus, anatomical connections in V1 and V2 support the notion of an asymmetric sorting of magnocellular and parvocellular contributions to MT and V4.

\section{Relationship between $P$ and $M$ pathways and the parietal and temporal cortical streams}

One hypothesis of the roles of parallel pathways in visual processing is that the $M$ and $P$ pathways establish two fundamental sensory domains that are analyzed in cortex more or less independently by the parietal and temporal streams of processing. This line of reasoning starts from the observation that the $M$ pathway deals with spatially coarse dynamic stimuli and ignores color and shading, while the $\mathrm{P}$ pathway deals with static information but preserves color, shading, and fine detail. The selectivities of the $M$ pathway appear to be well suited for visual motion processing in the parietal stream, while those of the $P$ 
pathway could support processing of color and shape in temporal cortex. The present data do not support the idea of a simple correspondence between the $\mathrm{P}$ and $\mathrm{M}$ subcortical pathways and the temporal and parietal streams of processing in extrastriate visual cortex. While MT is dominated by magnocellular inputs (Maunsell et al., 1990), neither parvocellular nor magnocellular inputs strongly dominate neuronal responses in either the superficial layers of V1 or in V4. There is every reason to expect that magnocellular and parvocellular contributions are comparably mixed at later stages of the temporal pathway. Thus, the relationship between the subcortical and cortical pathways appears to be neither a one-to-one mapping nor a complete intermixing, but instcad an asymmetric sorting.

Figure 10 summarizes our current view of magnocellular and parvocellular contributions to macaque visual cortex. While this diagram is undoubtedly incomplete and will need revision as our understanding improves, it indicates some of the complexity in the relationships between the subcortical pathways and the cortical streams of processing. Solid arrows represent magnocellular contributions and the dashed arrows represent parvocellular contributions (although the two types of signals are largely intermixed where both are present). It can be seen that magnocellular contributions enter the temporal stream of processing at its earliest stages in cortex. There is an established projection from layers $4 \mathrm{C} \alpha$ and $4 \mathrm{~B}$ to the blobs in V1 (Lachica et al., 1992). Magnocellular contributions are also seen in the interblobs (Nealey and Maunsell, 1994), but how they arrive there has not been established. Arrows connecting the V1 blobs and interblobs and the V2 thin stripes and interstripes represent a speculation that lateral connections within these areas (e.g., Rockland and Lund, 1983; Rockland, 1985) may be a route by which magnocellular contributions reach the interblobs and interstripes. It is also possible that a weak direct projection from layer $4 \mathrm{C} \alpha$ to the interblobs may exist.

Figure 10 also suggests that the magnocellular signals in V4 arrive primarily from V1 and V2, rather than by the connection with MT (Maunsell and Van Essen, 1983). This idea is supported by the observation that the effects of selective magnocellular LGN inactivation on the superficial layers of V1 (Nealey and Maunsell, 1994) closely resemble those in V4. Other routes could also bring $\mathrm{M}$ pathway signals to $\mathrm{V4}$, but this appears less likely for two reasons. First, inactivation of V1 abolishes responses in V4 (Girard et al., 1991), but not MT (Rodman et al., 1989; Girard et al., 1992), suggesting that most input to V4 arises from VI rather than MT. Second, the interconnections between MT and V4 may not provide a major avenue for intermixing because the substantial parvocellular presence in V4 is not apparent in the activity in MT (Maunsell et al., 1990). These connections may instead subserve a primarily modulatory function.

Because the $\mathrm{M}$ and $\mathrm{P}$ pathways are not related in a one-toone fashion to the parietal and temporal streams of processing we conclude that the distinctions between the subcortical pathways are not determined by the needs of these specific cortical streams of processing. It seems more likely that differences between the subcortical pathways represent an efficient method of transmitting low-level information, and that the cortical streams draw on information coded in the $\mathrm{P}$ and $\mathrm{M}$ pathways according to their particular needs. This view places a different emphasis on the role of the subcortical pathways, treating the different response properties of the $P$ and $M$ pathways less as fundamental perceptual domains and more like an efficient coding scheme

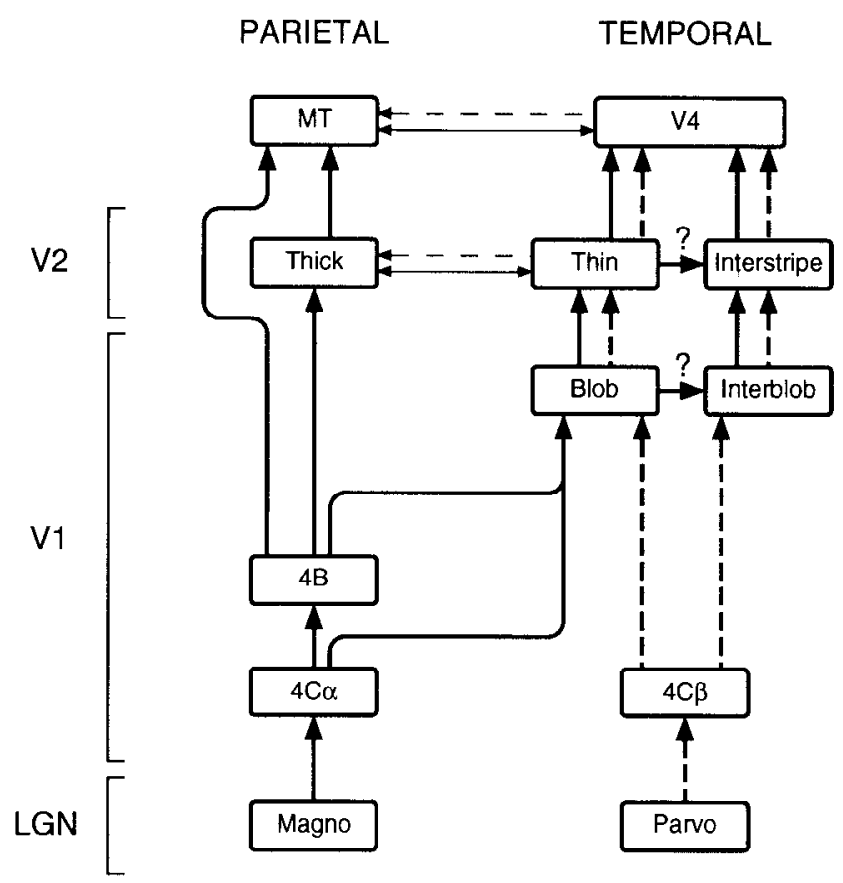

Figure 10. Relationships between the $\mathrm{M}$ and $\mathrm{P}$ pathways and the parietal and temporal streams of processing. A subset of the known components and connections is shown. Magnocellular contributions are indicated by solid arrows; parvocellular contributions are indicated by dashed arrows. MT is dominated by magnocellular inputs: the interconnections with V4 and between the thin and thick stripes in V2 do not appear to provide substantial parvocellular contributions (indicated by the thinness of those arrows). In contrast, magnocellular contributions enter the temporal stream as early as VI. Selective LGN inactivation suggests that magnocellular contributions are present in all of the major routes from $\mathrm{V} 1$ to $\mathrm{V} 4$. It is not known how magnocellular signals reach the interblobs and interstripes. The possible involvement of lateral connections in V1 and V2 is suggested by arrows marked by question marks.

for maximizing the information transmitted about different stimulus dimensions. If this is the case, then the asymmetric sorting of $\mathrm{M}$ and $\mathrm{P}$ pathway contributions to the parietal and temporal streams would reflect the information needed for the different types of behaviors mediated by the latter. The parietal pathway would depend largely on $M$ pathway contributions because the spatiotemporal and contrast response properties of the $M$ pathway are well suited for tasks like navigating in a complex three-dimensional environment, while the color or fine detail information of the $P$ pathway may be of little value. The temporal pathway, in contrast, would depend on the color, shading, and high spatial resolution of the $P$ pathway for recognizing objects, but may also benefit appreciably from the superior contrast response of the $M$ pathway. In line with this, recent evidence by Lee et al. (1993) suggests that the $M$ pathways make an important contribution to tasks that require high spatial resolution.

This view is consistent with results from recent behavioral studies. The behavioral deficits of animals with selective LGN lesions are best described in terms of the loss of low-level visual information. The higher visual functions associated with the cortical pathways generally remain intact. For example, lesions of the magnocellular LGN cause deficits in tasks that require the use of low spatial frequencies and high temporal frequencies, but leave acuity, motion discrimination, and stereopsis intact (Merigan and Maunsell, 1990; Merigan et al., 1991; Schiller et 
al., 1991a,b). Parvocellular lesions cause deficits in tasks that require the use of high spatial or low temporal frequencies or color, while leaving shape discrimination unaffected (Schiller et al., 1991 a,b; Lynch et al., 1992).

\section{References}

Baizer JS, Ungerleider LG, Desimone R (1991) Organization of visual inputs to inferior temporal and posterior parietal cortex in macaques. J Neurosci 11:168-190.

Blasdel GG, Lund JS, Fitzpatrick D (1985) Intrinsic connections of macaque striate cortex: axonal projections of cells outside lamina $4 \mathrm{C}$. J Neurosci 5:3350-3369.

Connolly M, Van Essen D (1984) The representation of the visual field in parvocellular and magnocellular layers of the lateral geniculate nucleus in the macaque monkey. J Comp Neurol 226:544-564.

Desimone R, Schein SJ (1987) Visual properties of neurons in area V4 of the macaque: sensitivity to stimulus form. J Neurophysiol 57 : $835-868$.

Desimone R, Ungerleider LG (1989) Neural mechanisms of visual processing in monkeys. In: Handbook of neuropsychology, Vol 2 (Boller F, Grafman J, eds), pp 267-299. New York: Elsevier.

DeYoe EA, Sesola LC (1991) Distincl pathways link anatomical subdivisions of V4 with V2 and temporal cortex. Soc Neurosci Abstr 17: 1282.

DeYoe EA, Van Essen DC (1985) Segregation of efferent connections and receptive field properties in visual area $V 2$ of the macaque. Nature 317:58-61

DeYoe EA, Van Essen DC (1988) Concurrent processing streams in monkey visual cortex. Trends Neurosci 11:219-226.

Felleman DJ, McClendon E (1991) Modular connections between area V4 and temporal lobe area PITv in macaque monkeys. Soc Neurosci Abstr 17:1282.

Felleman DJ, Van Essen DC (1991) Distributed hierarchical processing in the primate cerebral cortex. Cereb Cortex 1:1-47.

Ferrera VP, Nealey TA, Maunsell JHR (1992) Mixed parvocellular and magnocellular geniculate signals in visual area V4. Nature 358: $756-758$.

Fitzpatrick D, Lund JS, Blasdel GG (1985) Intrinsic connections of macaque striate cortex: afferent and efferent connections of lamina 4C. J Neurosci 5:3324-3349.

Gallyas F (1979) Silver staining of myelin by means of physical development. Neurol Res 1:203-209.

Girard P, Salin PA, Bullier J (1991) Visual activity in macaque area V4 depends on area 17 input. Neuroreport 2:81-84.

Girard P, Salin PA, Bullier J (1992) Response selectivity of neurons in areas MT of the macaque monkey during reversible inactivation of area V1. J Neurophysiol 67:1437-1446.

Lachica EA, Beck PD, Casagrande VA (1992) Parallel pathways in macaque monkey striate cortex: anatomically defined columns in layer III. Proc Natl Acad Sci USA 89:3566-3570.

Lee BB, Wehrhahn C, Westheimer G, Kremers J (1993) Macaque ganglion cell responses to stimuli that elicit hyperacuity in man: detection of small displacements. J Neurosci 13:1001-1009.

Livingstone MS, Hubel DH (1984) Anatomy and physiology of a color system in the primate visual cortex. J Neurosci 4:309-356.

Livingstone MS, Hubel DH (1987a) Psychophysical evidence for separate channels for the perception of form, color, movement, and depth. J Neurophysiol 7:3416-3468.

Livingstone MS, Hubel DH (1987b) Connections between layer 4B of area 17 and thick cytochrome oxidase stripes of area 18 in squirrel monkey. J Neurosci 7:3371-3377.

Livingstone MS, Hubel DH (1988) Segregation of form, color, movement, and depth: anatomy physiology, and perception. Science 240 : $740-749$.

Lund JS, Boothe RG (1975) Interlaminar connections and pyramidal neuron organisation in the visual cortex, area 17, of the macaque monkey. J Comp Neurol 159:305-334.

Lund JS, Lund RD, Hendrickson AE, Bunt AH, Fuchs AF (1976) The origin of efferent pathways from the primary visual cortex, area 17 , of the macaque monkey as shown by retrograde transport of horseradish peroxidase. J Comp Neurol 164:287-304.

Lund JS, Henry GH, MacQueen CL, Harvey AR (1979) Anatomical organization of the primary visual cortex (area 17) of the cat: a comparison with area 17 of the macaque monkey. J Comp Neurol 184: 599-618.

Malpeli JG, Schiller PH (1979) A method of reversible inactivation of small regions of brain tissue. J Neurosci Methods 1:143-151.

Malpeli JG, Schiller PH, Colby CL (1981) Response properties of single cells in monkey striate cortex during reversible inactivation of individual lateral geniculate laminae. J Neurophysiol 46:1102-1119.

Martin KAC (1988) From enzymes to visual perception: a bridge too far? Trends Neurusci 11:380-387.

Maunsell JHR (1987) Physiological evidence for two visual subsystems. In: Matters of intelligence (Vaina LM, ed), pp 59-87. Dordrecht: Reidel.

Maunsell JHR, Newsome WT (1987) Visual processing in monkey extrastriate cortex. Annu Rev Neurosci 10:363-401.

Maunsell JHR, Van Essen DC (1983) The connections of the middle temporal visual area in the macaque and its relationship to a hierarchy of cortical visual areas. J Neurosci 3:2563-2586

Maunsell JHR, Nealey TA, DePriest DD (1990) Magnocellular and parvocellular contributions to responses in the middle temporal visual area (MT) of the macaque monkey. J Neurosci 10:3323-3334.

Merigan WH, Maunsell JHR (1990) Macaque vision after magnocellular lateral geniculate lesions. Vis Neurosci 5:347-352.

Merigan WH, Maunsell JHR (1993) How parallel are the primate visual pathways? Annu Rev Neurosci 16:369-402.

Merigan WH, Byrne C, Maunsell JHR (1991) Does primate motion perception depend on the magnocellular pathway"? J Neurosci 11: 3422-3429.

Mishkin M, Ungerleider LG, Macko KA (1983) Object vision and spatial vision: two cortical pathways. Trends Neurosci 6:414-417.

Nealey TA, Maunsell JHR (1994) Magnocellular and parvocellular contributions to the responses of neurons in macaque striate cortex. J Neurosci 14:2069-2079.

Rockland KS (1985) A reticular pattern of intrinsic connections in primate areas V2 (area 18). J Comp Neurol 235:467-478.

Rockland KS, Lund JS (1983) Intrinsic laminar lattice connections in primate visual cortex. J Comp Neurol 216:303-318.

Rudman HR, Gross CG, Albright TD (1989) Afferent basis of visual response properties in area MT of the macaque. I. Effects of striate cortex removal. J Neurosci 9:2033-2050.

Schiller PH, Malpeli JG, Schein SJ (1979) Composition of geniculostriate input to superior colliculus of the rhesus monkey. J Neurophysiol 42:1124-1133.

Schiller PH, Logothetis NK, Charles ER (1991a) Functions of the colour-opponent and broad-band channels of the visual system. Nature 343:68-70.

Schiller PH, Logothetis NK, Charles ER (1991b) Role of the colouropponent and broad-band channels in vision. Vis Neurosci 5:321316.

Shipp S, Zeki S (1985) Segregation of pathways leading from area V2 to areas V4 and V5 of macaque monkey visual cortex. Nature 315: 322-325.

Ungerleider LG, Mishkin M (1982) Two cortical visual systems. In: Analysis of visual behavior (Ingle DJ, Goodale MA, Mansfield RJW, eds), pp 549-585. Cambridge, MA: MIT Press.

Van Essen DC (1985) Functional organization of primate visual cortex. In: Cerebral cortex (Peters A, Jones EG, eds), pp 259-329. New York: Plenum.

Van Essen DC, Maunsell JHR (1983) Hierarchical organization and functional streams in the visual cortex. Trends Neurosci 6:370-375.

Van Essen DC, Maunsell JHR, Bixby JL (1981) The middle temporal visual area in the macaque: myeloarchitecture, connections, functional properties and topographic organization. J Comp Neurol 199: 293-326.

Zeki SM (1971) Cortical projections from two prestriate areas in the monkey. Brain Res 19:63-75.

Zeki SM (1973) Colour coding in rhesus monkey prestriate cortex. Brain Res 53:422-427.

Zeki SM (1978a) The distribution of wavelength and orientation selective cells in different areas of monkey visual cortex. Proc R Soc Lond [Biol] 217:449-470.

Zeki SM (1978b) Uniformity and diversity of structure and function in rhesus monkey prestriate visual cortex. I Physiol (Lond) 277:273290. 